T. Fassaert; S. van Dulmen; F. Schellevis; J. Bensing.

Active listening in medical consultations: development of the Active Listening Observation Scale. (ALOS- 7 global).

Patient Education and Counseling: 2007, Vol. 68, nr. 3, p. 258-264

Postprint Version 1.0

Journal website $\quad$ http://dx.doi.org/10.1016/j.pec.2007.06.011

Pubmed link $\quad$ http://www.ncbi.nlm.nih.gov/sites/entrez?Db=pubmed\&Cmd=ShowDetailView \&TermToSearch=17689042\&ordinalpos=1\&itool=EntrezSystem2.PEntrez.Pubm ed.Pubmed_ResultsPanel.Pubmed_RVDocSum

DOI 10.1016/j.pec.2007.06.011

This is a NIVEL certified Post Print, more info at http://www.nivel.eu

\title{
Active listening in medical consultations: Development of the Active Listening Observation Scale (ALOS-global)
}

\author{
THIJS FASSAERT ${ }^{\mathrm{A}}$, SANDRA VAN DULMEN ${ }^{\mathrm{A}}$, FRANÇOIS SCHELLEVIS ${ }^{\mathrm{A}, \mathrm{B}}$ AND JOZIEN BENSING $^{\mathrm{A}, \mathrm{C}}$ \\ ${ }^{a}$ NIVEL (Netherlands Institute for Health Services Research), Utrecht, the Netherlands \\ ${ }^{\mathrm{b}}$ VU University Medical Centre, Department of General Practice, Amsterdam, the Netherlands \\ 'Utrecht University, Department of Health Psychology, Utrecht, the Netherlands \\ Corresponding author at: NIVEL, P.O. Box 1568, 3500 BN Utrecht, the Netherlands. Tel.: \\ +31302729703;fax: +31302729729.
}

\section{AbSTRACT}

Objective:Active listening is a prerequisite for a successful healthcare encounter, bearing potential therapeutic value especially in clinical situations that require no specific medical intervention. Although generally acknowledged as such, active listening has not been studied in depth. This paper describes the development of the Active Listening Observation Scale (ALOS-global), an observation instrument measuring active listening and its validation in a sample of general practice consultations for minor ailments.

Method : Five hundred and twenty-four videotaped general practice consultations involving minor ailments were observed with the ALOS-global. Hypotheses were tested to determine validity, incorporating patients' perception of GPs' affective performance, GPs' verbal attention, patients' self-reported anxiety level and gender differences.

Results:The final 7-item ALOS-global had acceptable inter- and intra-observer agreement. Factor analysis revealed one homogeneous dimension. The scalescore was positively related to verbal attention measured by RIAS, to patients' perception of GPs' performance and to their pre-visit anxiety level. Female GPs received higher active listening scores.

Conclusion:The results of this study are promising concerning the psychometric properties of the ALOS-global. More research is needed to confirm these preliminary findings.

Practice implications: After establishing how active listening differentiates between health professionals, the ALOS-global may become a valuable tool in feedback and training aimed at increasing listening skills.

\section{INTRODUCTION}

Consultations for minor ailments constitute a large part of the workload of general practitioners (GPs) [1] and [2]. Most minor ailments generally lack the need for professional medical input and are self-limiting. The reason why patients nevertheless consult their physician is likely to be associated with psychological 
T. Fassaert; S. van Dulmen; F. Schellevis; J. Bensing.

Active listening in medical consultations: development of the Active Listening Observation Scale. (ALOS- 7 global).

Patient Education and Counseling: 2007, Vol. 68, nr. 3, p. 258-264

factors, such as their fear of suffering from a serious condition [3]. In helping patients with minor ailments, GPs affective communication skills, such as active listening, may, therefore, in itself be therapeutic and ameliorative [4]. In addition, active listening may prevent unnecessary repeat visits, therefore, lowering GPs' workload [5] and [6].

Within western medical science, paying attention to the patient from a medical diagnostic point of view (i.e. physical examination) has since long been advocated at the expense of active listening necessary to learn more about the patient's suffering [7]. Yet, patients themselves seem to value the personal, active and listening doctor most [8]. Active listening can be understood as the necessary and first step towards patient centred healthcare that aims to unravel the reasons for visiting the physician from the perspective of the patient. Especially in the absence of serious physical symptoms, listening helps to understand patient's experience of illness [9] through recognizing and exploring patients' cues. Previous studies indeed show that active listening is associated with satisfaction [10] and with patient disclosure of specific concerns related to the complaints being presented [11].

Active listening is a patient-centred interview skill [12] that encompasses different elements, bearing instrumental and affective significance. On the one hand, active listening is an important instrument for gaining information, e.g. by the use of open ended questions, summaries and clarification [13]. On the other hand, it signifies the acknowledgement of a patient's suffering. What is more, the very act of listening assumes that there is something to listen to, i.e. that the patient has the opportunity to talk and express himself. Active listening, therefore, incorporates verbal as well as non-verbal facilitation of patient talk [14].

Even though the value of active listening has been generally acknowledged, it has almost been taken for granted considering the fact that it has not been studied in depth. So far, the concept has been primarily looked at in a qualitative or global way or using instruments that have either been contextualized in a nonmedical setting or capture verbal behaviour only [15]. This article describes the development and initial validation of an observation instrument, called the global Active Listening Observation Scale (ALOSglobal), in the context of consultations for minor ailments in general practice.

\section{METHODS}

\subsection{Subjects}

The present study was set within the Second Dutch National Survey of General Practice (DNSGP2), carried out in 2001 by NIVEL, the Netherlands Institute for Health Services Research [16]. During the video-observation part of DNSGP2, 142 GPs were observed by camera during consultations after receiving informed consent by the patient in question [17]. A fixed, unmanned video camera was set up in the consulting room directed at the GP on the front and at the patient from behind or the side. The privacy of the collected data was laid down in privacy regulations. Patient refusal rate was relatively low (11.9\%). Per GP, 15-20 consultations were recorded, resulting in 2784 video-observations of an equal number of different patients. Some patients attended the physician with a certain health problem for the first time, for others the recorded visit was a repeat visit. Health problems were coded using the International Classification of Primary Care (ICPC) [18]. For this study, only consultations with patients of 18 years and older consulting for minor ailments were selected. Minor ailments were defined as frequently presented and non-chronic health problems.

To meet the first criterion (i.e. 'frequently presented'), a consultation was included if a patient's most important health problem, as reported in a questionnaire filled out in the waiting room before the consultation, was common. Identification of patients' health problems within the DNSGP2 $(N=375,899$ patients) showed which health problems were presented most often in general practice [19]. Selected problems were related to ICPC-chapters D, L, R and S (digestive, musculoskeletal, respiratory and skin problems, respectively), and included problems such as stomach ache, low back pain without irradiation, cough and warts.

Following the second criterion, chronic health problems were excluded. Within DNSGP2, the GPregistration was episode oriented, and different consultations concerning the same health problem were linked to one episode. The final diagnosis made by the GP within one episode is considered to be the diagnosis of that episode. Consequently, the second criterion was met if the diagnosis corresponding to the 
T. Fassaert; S. van Dulmen; F. Schellevis; J. Bensing.

Active listening in medical consultations: development of the Active Listening Observation Scale. (ALOS-7 global).

Patient Education and Counseling: 2007, Vol. 68, nr. 3, p. 258-264

episode, which included the videotaped consultation did not indicate a chronic disease. For this purpose, a cluster of chronic diseases (e.g. migraine, asthma and diabetes) coded by ICPC was used [20].

Both criteria initially resulted in a selection of 574 videotaped consultations. During subsequent video observations, a number of patients appeared to present a chronic health problem, such as asthma. These consultations were marked $(N=50)$ and excluded afterwards. Consequently, 524 consultations remained for analysis.

\subsection{Procedure}

\subsubsection{Development}

In this study, active listening is understood as a communication strategy for recognizing and exploring patients' clues [9]. It (non)verbally stands for the acknowledgement of patient's suffering as well as the permission for the patient to speak. Individual items were selected that covered different aspects of these two dimensions as much as possible. Content validity of the ALOS-global was furthermore developed by intensive use of expert knowledge within NIVEL, as well as pilot observations. The initial scale consisted of 14 items (Table 1), measured on 5-point Likert-scales (end points labeled as 'never' to 'always'), measuring physicians' listening skills throughout the consultation. The ALOS-global was independently judged by two experts (a GP-counselor and a GP/epidemiologist), then slightly revised in order to achieve consensus about the content, and taken into observations. The first author (TF) was the main observer. A second observer from NIVEL viewed a selection of the videotapes to assess inter-observer reliability.

\section{[TABLE 1.]}

\subsubsection{Validation}

Validity was determined by testing four hypotheses. First, construct validity was assessed by correlating active listening and verbal attention measured by the Roter Interaction Analysis System (RIAS) [21]. RIAS verbal attention includes physicians' statements of empathy, as well as legitimizing (i.e. statements indicating that actions or emotions of other persons are understandable or normal) and partnership (i.e. statements that assure support). Consequently, a moderate positive correlation was expected.

Second, we expected patients' pre-visit state anxiety, measured by the State-Trait Anxiety Inventory (STAI) and active listening measured by the ALOS-global to be positively related, as active listening is thought to be especially indicated in response to another person's prevailing emotions [22]. Possible differences in communication between initial and follow-up consultations were taken into account [23]. The correlation between anxiety and active listening was expected to be stronger for initial consultations than for follow-up consultations, as multiple consultations for minor conditions have been found to be associated with a negative GP attitude, likely to result in being less responsive to emotions [24] and [25].

Third, the validity of the ALOS-global was assessed by looking at the association between active listening and the evaluation of the affective skills of the GP by the patients, using the affective performance subscale of the quality of care through the patients' eyes (QUOTE communication) [17].

Finally, gender differences were calculated between male and female GPs to evaluate discriminant validity [26]. Female GPs were expected to receive significantly higher scores on the ALOS-global than male GPs.

\subsection{Measures}

The patients completed a questionnaire before and immediately after each single consultation that was recorded of them. Among others, the questions asked for demographic characteristics (gender, age, education), the nature of patients' health problems, patients' level of anxiety before seeing the GP (STAI) and their evaluation of GPs' affective performance during the visit (QUOTE communication).

\subsubsection{STAI}

State anxiety was measured using the shortened Dutch version of the STAI [27] and [28]. This is a wellvalidated questionnaire, consisting of 10 items. Items are rated on 4-point answering scales $(1=$ 'not at all' to 4 = 'very much so'), and thus total scores range from 10 to 40 . Items ask respondents to state how they currently feel regarding statements that are worded either positively (e.g. 'I feel calm') or negatively (e.g. 'I feel strained'). During DNSGP2 the STAI was completed by patients right before and immediately after the 
T. Fassaert; S. van Dulmen; F. Schellevis; J. Bensing.

Active listening in medical consultations: development of the Active Listening Observation Scale. (ALOS- 7 global).

Patient Education and Counseling: 2007, Vol. 68, nr. 3, p. 258-264

consultation. Pre-consultation information on the STAI was missing for $19 \%$ of the patients. Cronbach's alpha was 0.90 .

\subsubsection{QUOTE communication}

The patient perceived performance by the GP was measured using the conceptual framework of the QUOTE [17]. Before the consultation, the patients reported how important they considered different aspects of communication for the forthcoming visit ( $1=$ 'not important' to $4=$ 'utmost important'). After the consultation, they rated whether the GP had performed these aspects ( $1=$ 'not' to 4 = 'yes'). Factor analysis of both the pre-visit and post-visit lists of questions on preference and performance revealed two relevant sub-scales: an affect-oriented scale of 7 communication aspects and a task-oriented scale of 6 communication aspects (Cronbach's alpha between 0.74 and 0.89). In the affect-oriented performance scale, the patients rated after the visit to what extent they have perceived their GP to have given enough attention; listened well; taken enough time; been friendly; been frank; taken the problem seriously; and been empathic. The sumscore indicates GPs' affective performance through the eyes of the patient.

\subsubsection{RIAS}

RIAS is a well-validated observational instrument measuring verbal communication behaviour in health care, used in various studies [21], [29], [30] and [31]. RIAS measures on several dimensions, applicable to both physician and patient, and was used to rate most consultations at an earlier stage of DNSGP2 [17]. The RIAS instrument discriminates between affective or socio-emotional utterances and instrumental or taskrelated utterances. Affective utterances concern statements needed to establish a therapeutically effective relationship between patient and physician (e.g. 'giving comfort', 'reassurance', 'showing optimism, concern and understanding'). RIAS data were available from $79 \%$ of the consultations. Pearson's $r$ indicated good results for inter-observer reliability (0.72-0.95 for GP-behaviour and $0.74-0.96$ for patientbehaviour).

So, for the purpose of validation, the patient's pre-visit anxiety score, the patient's perception of the GP's affective performance and the RIAS category verbal attention were used.

\subsection{Analysis}

First, the ALOS-global was judged on inter- and intra-observer reliability using Pearson's correlation coefficient $(r)$. A random sample of 20 consultations was selected and independently rated by two observers. A second random sample was used to determine intra-observer agreement. Items with slight agreement $(r<0.50)$ [32] were removed, and overall agreement (mean $r$ ) was calculated. Principal Component Analysis (PCA) with Varimax rotation was then used to study robustness of the scale. Factors with eigenvalues over 1 were extracted, if supported by the scree plot. Appropriateness of PCA was tested using the Kaiser-Meyer-Olkin (KMO) measure of sampling adequacy and Bartlett's test of sphericity. Items failing to load at least 0.40 on any factor were left out. Internal consistency and homogeneity were determined by calculating Cronbach's alpha. With respect to validation, Spearman's correlation coefficient for non-parametric correlations was used to measure associations with RIAS and STAI, as these tend to show non-normal distributions.

Statistical analyses were done using SPSS version 11.5. Correction for clustering (multilevel analysis) was not considered appropriate as the number of GPs, as well as the number of consultations per GP, were too small (139 GPs with on the average 3.8 patients each). Kreft argues that 150 groups with five observations within each group should be sufficient to obtain a power of 0.90 [33]. However, fewer observations (either groups or individuals) result in a rapid decline of power for the detection of cross-level interactions [34].

\section{RESULTS}

\subsection{Sample characteristics}

The sample consisted of 139 GPs, of whom 105 were male (75.5\%). Male GPs were on average 47.9 years old $( \pm 5.7)$, female GPs were younger $(44.1 \pm 7.0$ years) $(p<0.01)$. More than half of the GPs worked fulltime (51.1\%). Most GPs (38.8\%) worked in a group practice, while 31.7 and $29.5 \%$ of the GPs worked solo or with one partner, respectively. GPs were settled for at least 1 year and at most 32 years (average $15.6 \pm 8.3$ years). 
T. Fassaert; S. van Dulmen; F. Schellevis; J. Bensing.

Active listening in medical consultations: development of the Active Listening Observation Scale. (ALOS- 7 global).

Patient Education and Counseling: 2007, Vol. 68, nr. 3, p. 258-264

Two hundred and ninety-nine patients whose consultations were recorded (57.1\%) were female (Table 2). Mean age at the time of the consultation was 45.7 years $( \pm 16.6)$ for women and 46.5 years for men $( \pm 16.6)$ (n.s.). Sixty-three patients (12.0\%) presented a minor ailment related to the digestive system, while 270 (51.5\%), 130 (22.8\%) and 61 (11.6\%) minor ailments were related to the musculoskeletal system, the respiratory system and the skin, respectively. The patients visited their GP on average 1.3 times during the illness episode (range 1-4).

\section{[TABLE 2].}

\subsection{Inter- and intra-observer agreement}

Items 1, 4, 5, 6, 10 and 14 (Table 1) had poor inter-observer agreement $(r<0.50$; Cohen's kappa $<0.34$ ) and were, therefore, removed. Overall inter-observer agreement for the remaining eight items was moderately good (mean $r=0.60$, range $0.50-0.70$; mean kappa $=0.52$, range $0.43-0.62$ ), and so was the overall intra-observer agreement $(r=0.58$, range $0.51-0.62)$. The eight items were subjected to PCA.

\subsection{Dimensionality}

PCA was considered appropriate, as the Kaiser-Meyer-Olkin (KMO) measure of sampling adequacy was 0.87 . Bartlett's test of sphericity was highly significant $(p<0.01)$. PCA with Varimax-rotation resulted in a one-factor solution, with an eigenvalue of 3.8, explaining $47.5 \%$ of total variance (Table 3). Corrected item-total correlations were between 0.37 and 0.71 . Exclusion of the item with the lowest corrected itemtotal correlation (i.e. The GP spends time on social talk) resulted in a slight increase of Cronbach's alpha. This item was, therefore, removed as well. The final 7-item scale evidenced good internal consistency (Cronbach's alpha 0.84).

\section{[TABLE 3]}

\subsection{Item responses}

Table 4 shows the range of responses over the 7-item scale. Items of the ALOS-global predominantly revealed normal distributions. In the majority of the consultations, GPs showed not to be distracted throughout the consultation. Also, responses revealed that GPs are not hasty and give their patient time and space to present the problem. Asking exploring questions was less often observed as was GP's non-verbal expression of understanding.

\section{[TABLE 4.]}

\subsection{Validity}

There was a positive relationship between active listening (ALOS sumscore) and GPs' verbal attention measured by RIAS (Table 5). In addition, a small but significant positive relationship was found between GPs' active listening as rated by the observers and the patients' post-visit perception of GPs' affective performance measured with the QUOTE. Using the complete dataset, no significant relationship was found between patients' pre-consultation anxiety and GPs' active listening. However, when the whole set of consultations was broken down by rank order, i.e. initial and follow-up visits, the patients' pre-consultation anxiety did appear to be associated with more active listening during initial visits. With respect to GPs' verbal attention, the significant positive association with active listening had to be ascribed entirely to follow-up contacts. Finally, a difference was found between the ratings of male and female GPs, indicating that female GPs scored somewhat higher on active listening than their male counterparts, i.e. 24.8 and 23.5, respectively $(F=6.56, p=0.01)$.

\section{[TABLE 5.]}

\section{DISCUSSION AND CONCLUSION}


T. Fassaert; S. van Dulmen; F. Schellevis; J. Bensing. Active listening in medical consultations: development of the Active Listening Observation Scale. (ALOS- 7
global).

Patient Education and Counseling: 2007, Vol. 68, nr. 3, p. 258-264

\subsection{Discussion}

This article described the development of a new observation instrument aimed at measuring active listening in general practice, called ALOS-global, the global active listening by observation scale, and its initial validation in the context of consultations for minor ailments. Although the ALOS-global has been constructed for application in observation research, the individual items of the instrument may also provide useful starting-points for individual feedback and education in general practice.

With respect to reliability and validity of the ALOS-global, results are promising. There was a significant correlation between active listening measured by the ALOS-global and the RIAS category verbal attention. Although significant, the association was limited, which may be explained by the fact that both instruments measure different constructs to some extent. Beside statements reflecting attention, RIAS-category verbal attention also includes statements that indicate legitimizing or partnership [21].

Additionally, validity of the ALOS-global was supported by the finding that patients' anxiety levels shortly before the consultation were associated with GPs' active listening skills during the consultation, but this appeared to be the case only during initial visits. This confirmed our prior expectation that anxious or upset patients were more likely to elicit active behaviour in their GP. Possibly, GPs consider consultations for a new health problem more important for establishing a good atmosphere than follow-up consultations for the same health problem. Additional evidence exists that physicians in primary care often miss opportunities for affective communication, or may even choose not to pursue them [35].

Relationships differed by the rank number of the visit. This indicates that for research purposes the distinction between initial and follow-up consultations is useful and appropriate. For example, in consultations with patients with type 2 diabetes, van Dulmen et al. [23] found that internists showed more instrumental communication during initial consultations, as opposed to subsequent consultations in which affective communication played a more central role. Results from the present study suggest that anxiety increases active listening indeed, but only during initial visits. In addition, the stronger relationship between active listening and verbal attention during follow-up visits suggest that with new patients active listening encompasses more than verbal attention only. So far, only few observational studies have taken into account the possible differences in communication between initial and follow-up visit.

External validity of the results might be limited by the composition of the study sample. We aimed at selecting only patients presenting minor ailments for whom active listening is likely to be one of the relevant therapeutic tools. As chronic and more severe illnesses were excluded, it is unknown to what extent the results of this study are representative for the total general practice population. As a result, more effort is needed to determine validity of the instrument in other general practice consulters. Additionally, we do not exactly know how homogeneous our sample of minor ailment-patients was. In Section 2 , we reported the exclusion of 70 patients who appeared to present a non-minor ailment. We acknowledge the possibility that consultations for non-minor ailments were missed, possibly as a result of inadequate registration by the GP. On the other hand, we might have excluded chronically ill patients who presented a minor ailment that was not related to their chronic illness. One might argue that these patients actually do belong to our sample. The rationale of including minor ailments was largely based on the presumption that active listening could bear potential therapeutic value when no specific medical intervention is needed, which is especially so in case of minor ailments where self-care is more appropriate. But, of course, active listening has relevance for other complaints as well.

Our study did not intend to investigate the effectiveness of active listening in general practice. Especially in the case of minor ailments, we consider it worthwhile addressing this matter in future studies. As stated before, minor ailments account for a considerable share of all consultations in general practice, but tend to disappear spontaneously or as a result of adequate self-care [2]. Taking into account the fact that a positive patient-physician relationship has been shown to be a key factor for successful guided self-management, and that physicians' active listening is associated with such a relationship [35], active listening might be helpful in GPs' everyday management of minor ailments in their practice.

\subsection{Conclusion}

The results of this study are promising concerning the psychometric properties of the ALOS-global. More research is needed to confirm these preliminary findings.

\subsection{Practice implications}

After establishing how active listening differentiates between different health professionals, the ALOSglobal may become a valuable tool in feedback and training aimed at increasing listening skills. 
T. Fassaert; S. van Dulmen; F. Schellevis; J. Bensing.

Active listening in medical consultations: development of the Active Listening Observation Scale. (ALOS-7 global).

Patient Education and Counseling: 2007, Vol. 68, nr. 3, p. 258-264

\section{ACKNOWLEDGMENTS}

We would like to thank Liesbeth van der Jagt from the Dutch College of General Practitioners for her contribution to the development of the instrument. This study was supported by Grant number 42000015 from ZonMw, the Netherlands Organisation for Health Research and Development.

\section{TABLES}

Table 1

Items from the initial scale including operationalisations

The GP...

1. Uses inviting body language

A high score is indicated when the GP uses an open and interested body attitude, for example when he/she uses facial expressions (like frowning, raising eyebrows, smiling, in contrast to

2. Shows not to be distracted during the consultation yawning or staring), faces the patient and makes eye contact.

A high score is indicated if the GP is not distracted during the consultation. Distractions interrupt a conversation in a non-productive or disturbing manner, for example by the telephone (constantly) ringing, an assistant walking into the room, the GP staring at the computer screen or looking into the patient's file while ignoring the patient.

3. Is not off-hand or hasty

A high score is indicated when, for example, the GP adequately responds to patient's questions, does not talk at high speed or with a lack of silences in the conversation.

4. Is obviously relaxed and confident A high score is indicated if the GP is comfortable and confident. Signs of nervousness lead to a lower score, for example if the GP makes restless movements with head, arms, hands or legs, ticks/clicks with a pen, drums his fingers on the desk or produces uncomfortable silences.

5. Is not detached A low score is indicated when, for example, the GP greets the patient in an uninterested way or does not greet the patient at all, avoids eye-contact or makes irrelevant jokes all the time. A high score is indicated when, if necessary, the GP uses or avoids professional language or jargon.

6. Adjusts his/her language to that of the patient

7.listens attentively

8. Gives patient time and space to present the problem

A high score is indicated when, for example, the GP uses (comfortable) silences in the conversation to give the patient a chance to think or to elaborate, encourages the patient to clarify the problem, notices (non)verbal signs from the patient that indicate an emotions or summarizes the contents of the conversation.

A high score is indicated when, for example, the GP allows the patient to finish when he or she is talking and spends more than the usual time on the patient if necessary.

9. Uses exploring questions

10. Creates an open atmosphere during the conversation A high score is indicated when, for example, the GP uses exploning or open-ended questions, that do not invite any particular answer, but open up discussion or elicit a wide range of answers. A high score is indicated when the GP creates an open and accepting atmosphere for discussion by allowing the patient to express his- or herself fully and by giving the patient the chance to disagree. In case of conflict or disagreement, the GP resists the temptation to immediately intervene, but hears the patient out.

11. Spends time on social talk

A high score is indicated when, for example, the GP discusses or asks about (non-)medical issues regarding patient's social life (i.e. work, school, children, weather)

12. Is good in leading the conversation

A high score is indicated when the GP takes the initiative in the conversation (if necessary), sets an agenda in collaboration with the patient, involves the patient, paraphrases, repeats decisions/future appointments or evaluates the consultation.

13. Expresses understanding non-verbally

A high score is indicated when the GP acknowledges patient's emotions non-verbally by, for example, nodding, smiling, sympathising eye-contact, supportive humming or a pat on the shoulder.

14. Dilates verbally upon patient's feelings or emotions

A high score is indicated when the GP acknowledges patient's feelings and emotions verbally by, for example, naming them, asking the patient to clarify the feeling(s) or emotion(s). 
T. Fassaert; S. van Dulmen; F. Schellevis; J. Bensing.

Active listening in medical consultations: development of the Active Listening Observation Scale. (ALOS-7 global).

Patient Education and Counseling: 2007, Vol. 68, nr. 3, p. 258-264

Table 2

Patient characteristics minor ailments sample and larger study population

\begin{tabular}{lll}
\hline Patient characteristics & $\begin{array}{l}\text { Sample (\%), } \\
N=524\end{array}$ & $\begin{array}{l}\text { DNSGP2 (\%), } \\
\text { Sex }\end{array}$ \\
$\quad$ & & \\
$\quad$ Male & 42.9 & 41.2 \\
$\quad$ Female & 57.1 & 58.8 \\
Age (years) & & \\
18-44 & 50.8 & 42.9 \\
$45-64$ & 31.8 & 35.5 \\
65 and older & 17.4 & 21.6 \\
Education level & & \\
$\quad$ Low & 33.6 & 31.2 \\
$\quad$ Medium & 52.6 & 53.8 \\
High & 13.9 & 15.8 \\
Minor ailment related to ICPC-chapter & \\
$\quad$ Digestive & 12.0 & 5.9 \\
$\quad$ Musculoskeletal & 51.5 & 21.9 \\
Respiratory & 22.8 & 13.6 \\
$\quad$ Skin & 11.7 & 11.1 \\
Other & - & 47.5 \\
Mean number of visits (S.D.) & $1.31(0.63)$ & $1.33(0.62)$ \\
in complaint episode & & \\
\hline
\end{tabular}

Table 3

Factor loadings of the ALTOR items

\begin{tabular}{lll}
\hline ALTOR items & $\begin{array}{l}\text { Factor } \\
\text { loadings }\end{array}$ & $\begin{array}{l}\text { Cronbach's alpha } \\
\text { if item deleted }\end{array}$ \\
\hline $\begin{array}{l}\text { The GP... } \\
\begin{array}{l}\text { 1. Shows not to be distracted } \\
\quad \text { during the consultation }\end{array}\end{array}$ & 0.66 & 0.82 \\
2. Is not off-hand or hasty & 0.68 & 0.81 \\
3. Listens attentively & 0.82 & 0.79 \\
4. Gives patient time and space & 0.74 & 0.81 \\
$\quad$ to present the problem & & \\
5. Uses exploring questions & 0.69 & 0.81 \\
6. Spends time on social talk & 0.47 & 0.84 \\
7. Is good in leading the conversation & 0.71 & 0.81 \\
8. Expresses understanding non-verbally & 0.70 & 0.81 \\
Percentage explained variance & 47.5 & \\
Overall Cronbach's alpha & & 0.83 \\
\hline
\end{tabular}

Table 4

Item responses and missing values $(N=524)$

\begin{tabular}{|c|c|c|c|c|c|c|}
\hline The GP... & Never $(\%)$ & Rarely $(\%)$ & Sometimes (\%) & Frequently $(\%)$ & Always $(\%)$ & Missing values (\%) \\
\hline 1. Shows not to be distracted during the consultation & 1.0 & 7.1 & 30.5 & 37.8 & 23.3 & 0.4 \\
\hline 2. Is not off-hand or hasty & 1.1 & 8.8 & 33.4 & 39.3 & 17.0 & 0.4 \\
\hline 3. Listens attentively & 1.5 & 8.4 & 22.1 & 38.4 & 29.6 & 0 \\
\hline 4. Gives patient time and space to present the problem & 2.1 & 13.0 & 26.0 & 41.0 & 17.6 & 0.2 \\
\hline 5. Uses exploring questions & 6.5 & 34.0 & 30.7 & 23.5 & 5.0 & 0.4 \\
\hline 6. Is good in leading the conversation & 2.3 & 24.8 & 34.7 & 31.5 & 5.5 & 1.1 \\
\hline 7. Expresses understanding non-verbally & 9.2 & 26.9 & 27.7 & 30.0 & 6.1 & 0.2 \\
\hline
\end{tabular}


T. Fassaert; S. van Dulmen; F. Schellevis; J. Bensing.

Active listening in medical consultations: development of the Active Listening Observation Scale. (ALOS- 7 global).

Patient Education and Counseling: 2007, Vol. 68, nr. 3, p. 258-264

Table 5

Relationships between GPs' active listening skills, patients' perception of GPs' affective behaviour, GPs' verbal attention and patients' pre-visit anxiety; Spearman's correlations

\begin{tabular}{|c|c|c|c|}
\hline & \multicolumn{3}{|l|}{ Active listening } \\
\hline & Total sample $r(N)$ & Initial visit $r(N)$ & Follow-up visit $r(N)$ \\
\hline GPs' affective performance (QUOTE) perceived by patients & $0.11^{\circ}(502)$ & $0.10(245)$ & $0.11(254)$ \\
\hline GPs' verbal attention (RIAS) rated by observers & $0.19^{* *}(413)$ & $0.12(202)$ & $0.26^{* *}(208)$ \\
\hline Pre-visit anxiety (STAI) reported by patients & $0.09(427)$ & $0.20^{* *}(210)$ & $0.00(216)$ \\
\hline
\end{tabular}

Due to some missing values in visit data, numbers in the second and third column do not add up to the total numbers.

$p<0.05$.

$* 0.01$.

\section{REFERENCES}

[1] K. Hassell, Z. Whittington, J. Cantrill, F. Bates, A. Rogers and P. Noyce, Managing demand: transfer of management of self limiting conditions from general practice to community pharmacies, $\mathrm{Br}$ Med J 323 (2001), pp. 146-147.

[2] C. Morris, J. Cantrill and M. Weiss, GPs' attitudes to minor ailments, Fam Pract 18 (2001), pp. 581-585.

[3] S.M. Campbell and M.O. Roland, Why do people consult the doctor?, Fam Pract 13 (1996), pp. 75-83.

[4] E.J. Cassell, Diagnosing suffering: a perspective, Ann Intern Med 133 (1999), pp. 531-534.

[5] J. Bensing, K. Schreurs and A. De Rijk, The role of general practitioner's affective behaviour in medical encounters, Psychol Health 11 (1996), pp. 825-838.

[6] A.M. van Dulmen and J.M. Bensing, Health promoting effects of the physician-patient encounter, Psychol Health Med 7 (2002), pp. 289-300.

[7] S.W. Jackson, The listening healer in the history of psychological healing, Am J Psychiatry 149 (1992), pp. 1623-1632.

[8] S. Steine, A. Finset and E. Laerum, What is the most important for the patient in the meeting with a general practitioner?, Tidsskr Nor Laegeforen 30 (2000), pp. 349-353.

[9] F. Lang, M.R. Floyd and K.L. Beine, Clues to patients' explanations and concerns about their illnesses. A call for active listening, Arch Fam Med 9 (2000), pp. 222-227.[10] M.B. Wanzer, M. Booth-Butterfield and K. Gruber, Perceptions of health care providers' communication: relationships between patientcentered communication and satisfaction, Health Commun 16 (2004), pp. 363-383.

[11] L.S. Wissow, D.L. Roter and M.E. Wilson, Pediatrician interview style and mothers' disclosure of psychosocial issues, Pediatrics 93 (1994), pp. 289-295.

[12] M. Rimondini, L. Del Piccolo, C. Goss, M. Mazzi, M. Paccaloni and C. Zimmermann, Communication skills in psychiatry residents-How do they handle patient concerns?, Psychother Psychosom 75 (2006), pp. 161-169.

[13] M. Simpson, R. Buckman, M. Stewart, P. Maguire, M. Lipkin and D. Novack et al., Doctor-patient communication: the Toronto consensus statement, BMJ 303 (1991), pp. 1385-1387.

[14] K. Robertson, Active listening. More than just paying attention, Aust Fam Physician 34 (2005), pp. $1053-1055$.

[15] F. Lang, R. McCord, L. Harvill and D.S. Anderson, Communication assessment using the common ground instrument: psychometric properties, Fam Med 36 (2004), pp. 189-198.

[16] G.P. Westert, F.G. Schellevis, D.H. de Bakker, P.P. Groenewegen, J.M. Bensing and J. van der Zee, Monitoring health inequalities through general practice: the Second Dutch National Survey of General Practice, Eur J Public Health 15 (2005), pp. 59-65.

[17] In: A. van den Brink-Muinen, A.M. van Dulmen, F.G. Schellevis and J.M. Bensing, Editors, Second Dutch National Survey of General Practice. Focus on communication: doctor-patient communication in the Netherlands, NIVEL, Utrecht (2004)

[18] H. Lamberts and M. Wood, ICPC (International Classification of Primary Care), Oxford University Press, Oxford (1987).

[19] M.W. van der Linden, G.P. Westert, D.H. de Bakker and F.G. Schellevis, Second Dutch National Survey of General Practice. Complaints and illnesses in the population and in general practice, NIVEL, Utrecht (2004)

[20] C.J. Yzermans, G.A. Donker, J.J. Kerssens, A.J. Dirkzwager, R.J. Soeteman and P.M. ten Veen, Health problems of victims before and after disaster: a longitudinal study in general practice, Int J Epidemiol 34 (2005), pp. 820-826.

[21] D. Roter, The Roter method of interaction process analysis, The Johns Hopkins University, Baltimore (2001). 
T. Fassaert; S. van Dulmen; F. Schellevis; J. Bensing.

Active listening in medical consultations: development of the Active Listening Observation Scale. (ALOS-7 global).

Patient Education and Counseling: 2007, Vol. 68, nr. 3, p. 258-264

[22] H. Gleitman, Social Development. In: H. Gleitman, Editor, Basic Psychology, W.W. Norton \& Company, Inc, New York, London (1995), pp. 417-456.

[23] A.M. van Dulmen, P.F. Verhaak and H.J. Bilo, Shifts in doctor-patient communication during a series of outpatient consultations in non-insulin-dependent diabetes mellitus, Patient Educ Couns 30 (1997), pp. 227-237.

[24] A.M. Plass, D.R. Timmermans and G. van der Wal, Decreasing the number of consultations for minor illnesses of Turkish and Dutch inhabitants of a deprived area in The Netherlands: an intervention study, Fam Pract 22 (2005), pp. 51-57.

[25] T. Stokes, M. Dixon-Woods and R.K. McKinley, Ending the doctor-patient relationship in general practice: a proposed model, Fam Pract 21 (2004), pp. 507-514.

[26] A. Brink-Muinen, S. van Dulmen, V. Messerli-Rohrbach and J. Bensing, Do gender-dyads have different communication patterns? A comparative study in Western-European general practices, Patient Educ Couns 48 (2002), pp. 253-264.

[27] C. Spielberger, Manual for the State-Trait Anxiety Inventory (STAI), Consulting Psychologists Press, Palo Alto (1983).

[28] H. van der Ploeg, P. Defares and C. Spielberger, Manual of the Dutch version of the State-Trait Anxiety Inventory, Swets and Zeitlinger, Lisse (1980)

[29] L.M. Ong, M.R. Visser, I.P. Kruyver, J.M. Bensing, A. van den Brink-Muinen and J.M. Stouthard et al., The Roter Interaction Analysis System (RIAS) in oncological consultations: psychometric properties, Psychooncology 7 (1998), pp. 387-401.

[30] J.M. Bensing, D.L. Roter and R.L. Hulsman, Communication patterns of primary care physicians in the United States and the Netherlands, J Gen Intern Med 18 (2003), pp. 335-342.

[31] S. van Dulmen, M. Nubling and W. Langewitz, Doctor's responses to patients' concerns; an exploration of communication sequences in gynaecology, Epidemiol Psichiatr Soc 12 (2003), pp. 98-102.

[32] Kreft IGG. Are multilevel techniques necessary? An overview, including simulation studies, June 25, 1996. Available at:

http://www.sciencedirect.com/science?_ob=RedirectURL\&_method=externObjLink\&_locator=url\&_plusSig $\mathrm{n}=\% 2 \mathrm{~B} \&$ targetURL=http $\% 253 \mathrm{~A} \% 252 \overline{\mathrm{F}} \% 252 \mathrm{Fwww}$. calstatela.edu $\% 252 \mathrm{Ffaculty} \% 252 \mathrm{Fikreft} \% 25 \overline{\mathrm{F} q u a r t e}$ rly\%252Fquarterly.html [accessed January 8, 2006].

[33] T. Snijders and R. Bosker, Standard errors and sample sizes for two-level research, J Educ Stat 18 (1993), pp. 237-259.

[34] W. Levinson, R. Gorawara-Bhat and J. Lamb, A study of patient clues and physician responses in primary care and surgical settings, JAMA 284 (2000), pp. 1021-1027.

[35] A. Kennedy, L. Gask and A. Rogers, Training professionals to engage with and promote selfmanagement, Health Educ Res 20 (2005), pp. 567-578. 